\title{
Charting Spiritual Care in Digital Health: Analyses and Perspectives
}

\author{
Simon Peng-Keller
}

In this final contribution, the main threads of the present volume will be merged and will be brought together, and the prospects for practice and research will be examined. In a first step, I take up the historical approach outlined in the introduction. In order to deepen our understanding of current developments, I identify and describe the main driver behind it. Then, in a kind of relecture of the debate, I recapitulate some of the critical and controversial points, and I outline what I take to be the main areas of convergence and the undisputed insights of the contributions to this collection. The paper concludes with desiderata for new research and an outline of the future for the charting of spiritual care.

\section{The Genesis and Drivers of a New Chaplaincy Practice}

As pointed out in the introduction, charting healthcare chaplaincy in medical records is anything but new. As a professional practice, it had already been developed in the first part of the twentieth century. However, until the digital revolution, it remained a marginal phenomenon. With the rise of EMRs, the framework has changed completely and has opened up new possibilities and challenges for healthcare chaplaincy. Much more than a mere technical adjustment, the introduction of EMRs has had a considerable impact on spiritual care. The changes studied in this book are part of a much larger and ongoing process with as yet unknown consequences: namely, the digitalization of society and healthcare.

Nevertheless, it would precipitate to attribute the new charting of spiritual care solely to digitalization. As the contributions of this volume prove, other drivers have also facilitated the new methods of record keeping. At least three of them are clearly

\footnotetext{
S. Peng-Keller $(\bowtie)$

University of Zurich, Zurich, Switzerland

e-mail: simon.peng-keller@uzh.ch
} 
identifiable: the emergence of a new paradigm of healthcare chaplaincy; the development of interprofessional spiritual care; and remarkable changes in Western societies concerning the role of religion and spirituality in public spaces. For a more detailed understanding of the evolution examined here, it is worth taking a closer look at these three drivers as well.

(1) For many decades, clinical pastoral education has developed under the influence of the approach developed by Carl Rogers in the early 1940s. Rogers' nondirective counseling has been severely criticized but has until now proved to be helpful. However, it is now being challenged by a new paradigm: outcomeoriented chaplaincy (Hall et al. 2016). Its programmatic claim is reminiscent of a similar turn in psychotherapy, which was triggered by the emergence of effectiveness research and accompanied by heated debate of Klaus Grawe's insistence that psychotherapy must progress from "confession to profession" (Grawe et al. 1994) that resonates perfectly with the goals of outcome-oriented chaplaincy. However, the development of this approach was triggered mainly by financial pressure. With new public management and the ongoing economization of healthcare, cost-benefit considerations reached chaplaincy in the 1990s. At the Barnes-Jewish Hospital in St. Louis, this pressure led Arthur Lucas and his colleagues to develop a new concept of healthcare chaplaincy. The discovery that compassionate presence and purposefulness are not mutually exclusive was a key insight in the learning process described: "In our explorations, we found being present with patients can include informed intentionality." (Lucas $2001,4)$ The central argument for outcome-oriented chaplaincy is the inevitability of objectives and critical evaluation. Nondirective presence is appropriate only if it fits with the needs and wishes of patients; and so the patients' needs and wishes must be assessed. After the chaplain's visit, it is then necessary to check if it was helpful. Documenting is necessary for accountability and critical self-evaluation.

(2) While the development of outcome-oriented chaplaincy responds to external factors, especially to economic pressure, interprofessional spiritual care has its roots in twentieth-century reformist healthcare movements as well as in research on spirituality and health (Peng-Keller 2019a). Understood as a common task of all caregivers and volunteers, spiritual care has been, for instance, an essential part of the modern hospice movement since its beginnings in the 1960s. But the issue has been present in other areas of modern healthcare as well. In a groundbreaking resolution in 1984, the WHO recommended that the spiritual dimension should be included in all areas of healthcare. Even though this resolution was accepted only hesitantly, it marked a milestone in the development of interprofessional spiritual care which would begin to institutionalize itself in the 1990s with the establishment of new research positions, training programs, and scientific journals. In recent decades, the empirical literature on spirituality and religiosity has covered more and more areas of healthcare. During the same period, there has also been a gradual integration of spiritual care into curricula and healthcare practice. For example, according to the new learning objectives 
for medicine in Switzerland, students must learn to take the spiritual dimension into account within a psychosocial history. When doctors and other health professionals start to assess the spiritual concerns of their patients, the question inevitably arises how these concerns should be noted in the EMR.

(3) The third driver is no less complex, encompassing cultural and mental changes as well as the circulation of religions and spiritualities through globalization and migration. Roughly speaking, it encompasses the spiritual pluralization of Western societies, including a repositioning of organized religion in public life (Peng-Keller 2019b). In (post-)secular and ideologically heterogeneous societies, the status of faith-based chaplaincy in public institutions is controversial. Remarkably, the number of chaplains in the public sector as a whole has grown in recent years, although the public funding of the chaplaincy is contested in many places. Winnifred Fallers Sullivan, in a fine-tuned analysis of this development, has described the new type of chaplain as a "strangely necessary figure, religiously and legally speaking, in negotiating the public life of religion today," as someone who "operates at the intersection of the sacred and the secular, a broker responsible for ministering to the wandering souls of a globalized economy and a public harrowed by a politics of fear" (Sullivan 2014, x-xi).

It is no wonder that in the military and the prison system, the impact of the "new governance in religious affairs" has been particularly significant, as the public interest here is most urgent. In the healthcare sector, the interests are more mixed, and the developments more heterogeneous. Nevertheless, they are to be found in all of the countries and contexts studied above. Particularly telling is the case of Québec, which shows how healthcare chaplaincy has been reshaped in recent decades by changes in society - with consequences for clinical documentation. As we learn from the paper of Bélanger and colleagues, healthcare in Québec was largely Catholic until well into the twentieth century. This slowly began to change in the 1960s. For chaplains, changes within the church could be seen in their new professional title. The traditional designation in Québec "aumônier" was replaced in the 1970s by "agents de pastoral," not least because non-ordained theologians and religious had replaced priests as chaplains. In the 1990s, the term "intervenant(e)s en soins spirituels" was coined. It signified the fact that chaplaincy is not limited to pastoral ministry and religious care. This change was embedded in the reorientation of Québec's politics toward what would be called "open secularism." A report commissioned by the Québec government and written by the sociologist Gérard Bouchard and the philosopher Charles Taylor explains this concept as follows: "Open secularism recognizes the need for a neutral state - legislation and public institutions must not favor a religious or secular view. But it also recognizes the importance that many people attach to the spiritual dimension of life" (Bouchard and Taylor 2008, 140f.). With regard to healthcare, the report calls for a holistic approach that takes into account a person's biopsychosocial and spiritual dimensions. Since a decree issued by the Ministry of Health in 2010, the responsibility for the provision and funding of healthcare chaplaincy lain with the health institutions themselves, which were given the legal obligation to attend to patients' "spiritual 
needs." Almost exactly the same story, but in a protestant version, could be told about healthcare chaplaincy in the Swiss canton of Vaud. Here as in Québec, the new regulation of the relationship between the state and the churches led to a repositioning of healthcare chaplaincy, a change which also manifested itself in an obligation to keep records.

In sum, the drivers of the rapid development of charting spiritual care in EMRs were manifold. Their complex interactions explain not only its speed but also the heterogeneity of current models and the tensions between the distinct goals of charting chaplaincy work in EMRs. For understandable reasons, these tensions are played down by those who advocate the implementation of this practice. This is one reason to explore them more closely in the following section.

\section{Convergences and Controversies}

In current discussions about the who, what, how, and why of recording spiritual care in EMRs, there is a growing convergence on at least four points. First, used as a tool for planning, coordination, and critical self-evaluation, appropriate forms of digital charting can benefit the work of chaplains. Second, it can also have undesired side effects. Third, any future healthcare chaplaincy will have to be a part of the evolving process of digital recording. In a highly specialized professional world where communication and recording is increasingly formed by digital tools, being absent from these new spaces would marginalize the work of chaplains even more. It would mean assimilating the role of chaplains to that of visiting ministers for community members. At least for those who are committed to the project of the clinical pastoral education, there is no alternative to the ongoing process of professionalization which includes standards for recording. Fourth, the ongoing change in healthcare and society, outlined above, forces the chaplaincy to become clearer about its nature and role.

At this point, the controversies begin. Some of them are not new but were already part of the CPE of the twentieth century. Focusing on the who of recording, the question of professional identity arises. The sensitive issue here is the proximity of chaplaincy work to psychological counseling and its language. If professional autonomy is to be preserved, distinct profiles are needed. But how can this be guaranteed if the core dimension of spiritual care characterized by particular spiritual traditions and affiliations recedes into the background? The paradigm of outcomeoriented chaplaincy may improve the self-reflectivity and the efficiency of spiritual caregivers, but it can't solve the underlying problem of professional identity, which is exacerbated by the current spiritual-religious diversification. The preceding remarks also seem to touch on matters of identity - and not just practical matters. Who and what are healthcare chaplains in the community of caregivers and in the context of interprofessional spiritual care? If they are themselves healthcare professionals, are they representatives of specific faith communities and spiritual traditions as well? Should they be active members of particular faith communities or rather active members in non-denominational professional associations (or both)? Is 
it necessary for their work that they are themselves believers and religious practitioners? How important is theological education for the identity of healthcare chaplains? Why not train psychologists in spiritual care so that they can fulfill the role of specialized spiritual carer?

The current development is quite paradoxical: In order to achieve a better professional status in healthcare, one clearly distinguishable from that of the psychologist, chaplains must avoid being exclusively health professionals. What gives chaplains a distinctive profile lies beyond secular healthcare. Thus, they are creatures of two worlds: the world of healthcare, with its rules and quality measures, and the world of a specific spiritual community, with its own language and practices. The first affiliation legitimizes the access chaplains have to the EMR, but it is the second affiliation that affords them their identity. How does this dual affiliation relate to the challenge of charting spiritual care in EMRs? As mentioned in the introduction to this volume, Richard Cabot and Russell Dicks have shown how professional and spiritual aspects are closely intertwined in pastoral documentation. On one level, charting spiritual care is a professional practice with strict rules and determining contexts. However, on a deeper level, the same activity can be understood and cultivated as a spiritual exercise. Cabot and Dicks particularly emphasize the creative side of writing: how the process of documenting can not only clarify an issue but inspire new ideas for future practice.

Thus, the questions of the what and the how of charting spiritual care are closely connected with the issue of professional identity. If "code switching" is essential for today's healthcare chaplains, as Wendy Cadge (2012) claims, then the practical knowledge of charting is entangled with the orientational knowledge stemming from the perspective of a spiritual view on life and death. As spiritual identities are formed by the language learned in one tradition or another, healthcare chaplaincy has to nourish itself with these languages while navigating with digital skills and codes. For clinical usage in secular and multi-faith contexts, standardized records are indispensable. Preformulated items broad enough to encompass a plurality of spiritual/religious beliefs, practices, and experiences might be a good compromise. However, the flipside of openness is vagueness, something contradictory to the goal of integrating orientational knowledge, the spiritual reframing of the situation of illness, into the charts. So, this task is perhaps best achieved in a free-text section. Narrative entries would serve this purpose best. Chaplains are "bearers of stories" (Anne Vandenhoeck), and theology is accustomed to raising its issues through narrative. But doesn't that also hold true for psychology?

The task of charting spiritual care into EMRs might be seen as a merely technical duty. However, with its questions of the who, the what, and the how, it touches the heart of chaplaincy as a spiritual profession in healthcare. If chaplains have their own way of being "bearers of stories" and of letting "stories breathe" (Frank 2010), then this is because of the shared stories that form their identities. They welcome the stories of the patients in a distinctive horizon, a specific web of significance. The narrow free-text spaces in EMRs might not be the best place for vibrant stories received and empowered within such horizons and webs. But they might at least offer opportunities for hints toward complementary sources of meaning, healing, and support. 


\section{Perspectives for Further Research}

The present volume documents the first two decades of charting spiritual care in EMRs as well as the first decade of research on it. On both levels we are concerned with pioneering work. The experimental and tentative character typical of emerging practices is often reflected in the corresponding research. Being involved in pioneering work is exciting but also challenging. It means navigating a changing field with few reliable landmarks. In this situation, clear strategies for research are all the more urgent. The available literature together with the papers collected here suggests six pathways:

\subsection{Empirical Evidence of the Impact of Charting}

The first priority for future research should be to investigate the impact of chaplaincy records on caregiving. Given the amount of time and energy it takes to develop and implement new tools and practices, it is essential to prove their appropriateness and usability. On the most general level, this concerns the tools themselves: Are they suitable for the declared aims? Are the goals named attained? And what unintended side effects are there? On a more specific level, the degree and way of implementation of a tool might be examined. If a practice doesn't reach its goals, the problem might lie with the technical tools. But it might be a lack of implementation, training, or supervision. Finally, one might focus on the impact of digital charting on interprofessional communication and contact with patients. Are the chaplaincy entries actually read? Are they understood and perceived as helpful? Do they make a difference to work at the bedside? Empirical evidence on the impact of charting spiritual care may also increase awareness of the potential of a digital practice as well as its limitations.

\subsection{Clarifying the Conceptual Framework}

This volume has also touched upon research into the conceptual framework of healthcare chaplaincy. As has been shown, the choice of tools and practices of charting always reflects a particular understanding of chaplaincy and spiritual care. Questions around divergent concepts of healthcare chaplaincy lie at a deeper level and call for independent discussion. But, as the development of digital charting tools and practices is shaped by these concepts, it is also necessary to address such questions in this context. Moreover, the effort to clarify the rationale for the participation of chaplains in medical record keeping may shed light on the broader discussion of interprofessional spiritual care. Or, as Bélanger and his colleagues put it, the practice of writing notes in EMR may force us to rethink the theological framework 
of healthcare chaplaincy. The conceptual decisions, especially, have a legal impact. What legitimizes (or even urges) the chaplaincy to participate in medical recording? If the claim to legitimacy is based on the assumption that the chaplaincy participates in medical care and shares its goals (e.g., pain relief), this has to be justified theologically as well.

\subsection{Hermeneutic Analysis of Professional Documentation}

The development of digital charting in chaplaincy has brought a neglected topic to the fore: the art of professional documentation in chaplaincy. From a research perspective, hermeneutical, narratological, and technical questions are at stake. Generally speaking, notes in EMR are a specific form of testimony. By means of a highly interpretative process, experiences are transformed into standardized text. This process could and should be studied with the aid of concrete examples. However, the question must also be discussed at a fundamental level: If the professional identity of chaplaincy lies, as is often claimed, in a specific perspective, namely, in the theological view of human life, then this perspective should be recognizable in the digital records of chaplains and in the categories of the tool used. What are the hallmarks of a theological hermeneutic for "living human documents" that can guide the practice of digital charting?

\subsection{Ethical Investigations}

Until now, ethical investigations concerning the charting of spiritual care have focused mainly on the issue of confidentiality. The discussion of this issue must take account of the different distinctions that are drawn in the various denominational traditions. Catholic theology, for instance, differentiates between the canonically regulated confessional secret and pastoral confidentiality, which are less clearly defined. Both in turn differ from legally regulated professional secrecy. The question of professional identity returns at this stage: As a pastoral profession, chaplaincy is regulated by the concepts and rules of a specific spiritual tradition; as profession in healthcare, the ethical standards and obligations of healthcare professionals are to be applied. Clarifications of these distinctions and overlaps may touch on the deeper question of the relevance and limits of confidentiality in interprofessional spiritual care. Furthermore, as Guy Jobin's contribution to this volume indicates, the charting of spiritual care calls for ethical reflection with respect to a number of different issues and not just confidentiality. Apart from the ubiquitous problem of data security, at least four further questions are to be addressed: What is the impact of technology on care and clinical judgment? How does charting affect the care relationship? Who is accountable for writing and reading records in 
interprofessional spiritual care? And finally, (how) is the aim of a common culture of values or a cross-professional ethos attainable?

\subsection{Charting Spiritual Care in the Context of Digital Health}

The question of the inherent dynamics of digitalization in healthcare forms a further research area. Just as digital tools transform the daily practice of physicians and nurses, so they also have profound implications for spiritual care, influencing how chaplains relate to patients, colleagues, and other caregivers. Digital tools shape the organization of work, the forms of communication, the patient's expectations, as well as the infrastructure and the atmosphere of healthcare institutions. As digitalization amounts to a profound transformation of the professional identity of healthcare professionals and chaplains, the changes it brings about must be studied in detail. How will the chaplain's offline ministry be changed and extended by new online spaces for (recording) spiritual care? At a more technical level, there is also the need for more intensive cooperation between IT researchers and practitioners in order to improve the digital tools and their usage.

\subsection{Digital Records as a New Tool of Chaplaincy Research}

Finally, recording spiritual care in EMRs opens new possibilities for research on spiritual care. As Paul Galchutt and Judy Connolly demonstrate in their contribution, analyzing the digital notes of chaplains can be not only very instructive but also participatory. Apart from the possibility of checking the adequacy as well as the impact of a certain kind of charting, the analysis of digital records with the methods of the digital humanities could provide precious information on the needs of patients, the work of chaplains, and the functioning of interprofessional collaboration. In view of the manifold possibilities which digitized data already offers for studies of all kinds, it is not unlikely that EMRs will be the central instrument for spiritual care research in the future.

\section{Outlining the Future}

Powered by the three drivers named at the beginning, healthcare chaplaincy is currently surfing through a "sea change" (Massey 2015). The new practice of recording spiritual care in EMRs reflects this change and is itself a catalyst of it. The medium shapes the message as well as the identities of those who use it. This is true for professionals, patients, and relatives. Digital communication between healthcare professionals and patients will intensify in the coming years. Just as telemedicine and 
cross-clinic EMRs will be interwoven in the future, so may chaplaincy documentation be increasingly intertwined with forms of telechaplaincy (as it has been introduced, for instance, by the influential US Department of Veterans Affairs). "Synthetic situations," in which online and offline spaces overlap (Knorr Cetina 2009), will be increasingly common for healthcare chaplaincy. While it is unlikely that telechaplaincy will replace the direct encounter in the sickroom, it is conceivable that in the future contact will be established digitally. It goes without saying that telechaplaincy, if part of the therapeutic concept, will also have to be recorded in EMRs.

Considering the current trends, the inclusion of interprofessional spiritual care in medical care is likely to become more widely established in the future. To the extent that chaplains participate in the medical treatment mandate, they are then to be regarded as health professionals and subjected to the same obligations. However, this is not a conclusive answer to the question of their professional identity as specialized spiritual caregivers. If spirituality has to do with transcendence and ultimate values, and if these are cultivated by distinct traditions and communities, then the identity of chaplains is a matter of their spiritual aim, of the ability to help patients navigate liminal spaces: in the realm of between life and death, between a meaningful cosmos and its collapse, and not least in the border zones between established faith communities and the individual's search for meaning. For these reasons, the "spiritual concerns" of patients and relatives must be afforded space in the online reality of EMRs.

\section{References}

Bouchard, Gérard, and Charles Taylor. 2008. Fonder l'avenir. Le temps de la conciliation. Rapport de la Commission de consultation sur les pratiques d'accommodement reliées aux différences culturelles. Québec. www.mce.gouv.qc.ca/publications/CCPARDC/rapport-finalabrege-fr.pdf.

Cadge, Wendy. 2012. Paging god. Religion in the Halls of medicine. Chicago: University of Chicago Press.

Frank, Arthur W. 2010. Letting stories breathe. A socio-narratology. Chicago: University of Chicago Press.

Grawe, Klaus, Ruth Donati, and Friederike Bernauer. 1994. Psychotherapie im Wandel. Von der Konfession zur Profession. Göttingen: Hogrefe.

Hall, Eric J., George H. Handzo, and Kevin Massey. 2016. Time to move forward. A new model of spiritual care to enhance the delivery of outcomes and value in health care settings, New York. https://spiritualcareassociation.org/docs/resources/time_to_move_forward_ report_2016-06-07.pdf.

Knorr Cetina, Karin. 2009. The synthetic situation: Interactionism for a global world. Symbolic Interaction 32 (1): 61-87.

Lucas, Arthur. 2001. Introduction to The discipline for pastoral care giving. Journal of Health Care Chaplaincy 10: 1-33.

Massey, Kevin. 2015. Surfing through a sea change: The coming transformation of chaplaincy training. Practice: Formation and Supervision in Ministry 35: 144-152.

Peng-Keller, Simon. 2019a. Spiritual Care im Gesundheitswesen des 20. Jahrhunderts. Von der sozialen Medizin zur WHO-Diskussion um die «spirituelle Dimension〉. In Spiritual Care im 
globalisierten Gesundheitswesen. Historische Hintergründe und aktuelle Entwicklungen, ed. Simon Peng-Keller and David Neuhold, 13-71. Darmstadt: Wissenschaftliche Buchgesellschaft Darmstadt.

2019b. Genealogies of 'spirituality'. An historical analysis of a travelling term. Journal for the Study of Spirituality 9 (2): 86-98.

Sullivan, Winnifred F. 2014. A ministry of presence. Chaplaincy, spiritual care, and the law. Chicago: University of Chicago Press.

Open Access This chapter is licensed under the terms of the Creative Commons Attribution 4.0 International License (http://creativecommons.org/licenses/by/4.0/), which permits use, sharing, adaptation, distribution and reproduction in any medium or format, as long as you give appropriate credit to the original author(s) and the source, provide a link to the Creative Commons license and indicate if changes were made.

The images or other third party material in this chapter are included in the chapter's Creative Commons license, unless indicated otherwise in a credit line to the material. If material is not included in the chapter's Creative Commons license and your intended use is not permitted by statutory regulation or exceeds the permitted use, you will need to obtain permission directly from the copyright holder.

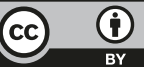

